\title{
Ineffectiveness of covert rehearsal with tones
}

\author{
ZEHRA F. PEYNIRCIOĞLU and MICHELLE L. PALMER \\ The American University, Washington, D.C.
}

\begin{abstract}
We investigated whether covert rehearsal of simple musical tones enhanced memory for those tones. Subjects were presented with a series of pairs (Experiment 1) or triples (Experiment 2) of tones played separately. Some of those pairs or triples were cued for covert rehearsal during a silent interval. Although both rehearsed and unrehearsed target items were better recognized than were lure items, rehearsed items were not recognized better than were unrehearsed items. Experiment 3 showed that repetition of such items in the presentation list did benefit memory, however. Twice-presented pairs of tones were better recognized than were once-presented pairs, which in turn were better recognized than were lure pairs.
\end{abstract}

We know that memory for verbal and pictorial materials can benefit from covert rehearsal (e.g., Graefe \& Watkins, 1980; Johnson, 1980). That is, thinking about such materials when they are no longer present improves memory performance compared to studying these materials only while they are physically present.

Research on whether nonverbal auditory materials can be rehearsed has been relatively sparse, however. Watkins and Peynircioglu (1982) presented three-word utterances and asked subjects to rehearse some of these along with the voice that uttered them; at test, the same words were uttered by many different voices and the task was to pick the original utterance. Rehearsal was effective and rehearsed utterances were more likely to be correctly recognized. That is, the act of concentrating on selected utterances and replaying them over and over in their minds enhanced the subjects' memory for these utterances. Similarly, in an unpublished study, Watkins and Peynircioğlu (1981) showed effective rehearsal for natural sounds such as the sound of thunder, a baby crying, or a dentist's drill.

This study investigated whether simple musical tones can also be effectively rehearsed by subjects who do not have absolute pitch and thus cannot make use of verbal labels for those tones. Recent research has shown that tones can be imaged and such mental imagery enhances immediate perceptual and cognitive tasks (e.g., Farah \& Smith, 1983; Halpern, 1988; Hubbard \& Stoeckig, 1988). The purpose of this study is to determine whether such mental manipulation can benefit longer term memory for tones.

\section{EXPERIMENT 1}

In this experiment, subjects were presented with a short series of tone pairs, with the tones in a pair presented sequentially, and asked to rehearse one of the pairs for the

We thank Beth Loots for her help in Experiment 2. Correspondence concerning this article should be addressed to Z. F. Peynircioglu, Department of Psychology, The American University, Washington, DC 20016. duration of the silent interval that followed. Of interest was whether in the subsequent recognition test the presented tone pairs would be discriminated from lure tone pairs that had not been presented and, more importantly, whether the rehearsed pairs would be more likely to be discriminated than would the simply "heard" pairs.

\section{Method}

Subjects. The subjects were 24 students at The American University who participated for extra credit in psychology classes. None reported having hearing problems or absolute pitch. Although not intentionally recruited as such, half of the subjects turned out to have had no musical training and the other half at least 1 year of training (ranging from 1 to 14 years, with a mean of 6.5 years).

Materials and Design. The stimuli were 12 pairs of successive tones that ranged from $A$ below middle $C$ to $A$ two octaves above. The lower note of the pair always came first in the succession, and the pairs were created in such a way that some interval relations were replicated. These 12 pairs were randomly divided into three sets (Sets A, B, and C) of four pairs each, with the constraint that each set included no more than one of each type of interval. In fact, there were two intervals common to Sets $A$ and $B$, two common to Sets $A$ and $C$, and one common to Sets B and C. Also, at least one note appeared twice in the same set and at least two notes were shared by two sets. Thus, remembering simply the interval or the pitch would not be as helpful as remembering the combination. One set served as the to-be-rehearsed items, another set as the only-to-be-heard items, and the third set as the lure items during the test phase. The sets were systematically rotated across subjects so that each set served in each condition equally often.

All pairs were first recorded onto Maxell tapes using a Casio MT220 electronic keyboard and a portable Sony dual-cassette tape recorder. Then, three presentation lists were created, each with a different combination of sets (Sets A and B, Sets B and C, and Sets A and C). In all presentation lists, the pairs of tones were presented in blocks of two. The two pairs in a block were separated by $2 \mathrm{sec}$, and the blocks were separated by $12 \mathrm{sec}$ (the rehearsal interval). After the two pairs were played, one pair was cued for rehearsal. The presentation order was the same for all subjects, but for half the subjects receiving each presentation list the first pair was cued for rehearsal (by raising a card with a " 1 " written on it) after Blocks 1 and 3 and the second pair was cued for rehearsal (by raising a card with a " 2 " written on it) after Blocks 2 and 4 . For the other half of the subjects, the cuing order was reversed. Since the subjects did not know how many blocks would be presented, they could not predict which pair would be cued for rehearsal after any given block.

The free-choice recognition test that followed comprised all 12 tone pairs presented at 8-sec intervals; the pairs were presented in the same order to all subjects and were randomly ordered with respect to whether they were target or lure items and, within the target items, whether they 
were rehearsed or only-heard items. The subjects were given blank sheets of paper to record their "ease of rehearsal" ratings for each item during the presentation phase and their responses during the test phase. A questionnaire asking for musical background was also given.

Procedure. The subjects were tested individually. They were told that they would be given two pairs of successive tones one after another and then they would be asked to rehearse one of these pairs. If the card the experimenter raised had a " 1 " written on it, they were to rehearse the first pair they heard, and if it had a " 2 ," the second pair. They were told that by rehearsal we meant that they should keep the two tones alive in their minds, to imagine them being played over and over again just as they had been played for the duration of the silent interval. Also, to make sure that only the to-be-rehearsed pair of tones actually got rehearsed, the subjects were told that the purpose of the other pair was to distract them and that they should try hard to not let their minds wander to the uncued pair since only the cued pairs would be included in the recognition test. Furthermore, they were asked to rate on a 3-point scale ( 1 corresponding to very hard and 3 to very easy) how easy it was to sustain concentration and rehearse the cued tones during each rehearsal interval.

Before starting with the presentation list, to clarify the rehearsal instructions and the procedure in general, the subjects were walked through a practice trial that consisted of two pairs of tones (but, unlike in the experiment proper, they were tested only on the rehearsed pair during practice). Also, since there would be no talking once the experiment started, the bulk of the test instructions were given before the presentation list. The part about also trying to recognize the "uncued" pairs, along with a short summary of the complete test instructions, was given to the subjects in writing after the presentation list was over.

After all eight pairs of tones had been presented and four of those pairs rehearsed, the subjects were given the recognition test. They were told (in writing) that of the tone pairs that would shortly follow, some were ones they had rehearsed, some were ones they had only heard, and others ones that had not been presented in the study list. Their task was to write yes or no after each test pair to indicate whether they recognized the tone pair as having been presented in the presentation list and also to give a confidence rating between 1 (guess) and 3 (sure) for each decision. After the recognition test was over, the subjects were given a questionnaire about their musical background.

\section{Results}

Two types of results are reported: Percentage of yes responses and more sensitive recognition ratings. The recognition ratings were obtained by combining the yes-no responses and the subjects' confidence ratings into a continuous 6-point scale by assigning no-3 responses (corresponding to It was not presented and I am sure) a weight of 1 and yes-3 responses (corresponding to It was presented and I am sure) a weight of 6 ; no-2, no-1, yes-1, and yes -2 responses were assigned weights of $2,3,4$, and 5, respectively. The results (along with those of Experiments 2 and 3 ) are presented in Table 1.

As can be seen, presentation had an effect. Although false-alarm rates were somewhat high, the subjects recognized presented pairs, regardless of whether they had been rehearsed or only heard, more often than lure items $[t(23)=$ $3.95, p<.01$ ]; also, even though the subjects were reluctant to give high confidence ratings when they said an item had been on the presentation list, overall, their recognition ratings were higher for such items than for lure items $[t(23)=3.43, p<.01]$. The same results were also obtained when rehearsed and only-heard items were compared with lure items separately $[\operatorname{ts}(23)=2.89$ and 3.70 for percentage of yes responses and $t \mathrm{~s}(23)=2.83$ and 3.11 for recognition ratings (all $p s<.01$ ), respectively]. There were no differences between the musically trained and naive subjects in their patterns of responses.

Of greater interest was the effect of rehearsal. There appeared to be no beneficial effect of rehearsing the pairs over simply hearing them. In fact, only-heard items were slightly, though insignificantly ( $p s>.10$ ), better recognized than were rehearsed items. There were no differences between the rehearsed pairs as a function of whether they were played first or second in a given block (we checked position in a block to see if the sound of the second pair impaired the rehearsability of the first pair by perhaps damaging echoic memory for the first pair). There were also no correlations between ease of rehearsal ratings given during study and actual recognition of those items during the test (as gauged by either measure); those items the subjects thought easier to concentrate on and keep mentally alive were not any more likely to be remembered than were those that were harder. Thus, whatever the subjects were doing during the rehearsal interval was not predictive of subsequent memory performance. Moreover, there were no differences between musically trained and naive subjects in their general patterns of responses; in fact, somewhat ironically, the difference in the recognition ratings between only-heard and rehearsed items approached significance $[t(11)=1.92, p<.10]$ for musically trained subjects in favor of only-heard items. Thus, spending an extra $12 \mathrm{sec}$ imaging a given pair of tones did not enhance its subsequent recognition.

Table 1

Percentage of Yes Responses and Recognition Ratings on a 6-Point Scale in Each Experiment

\begin{tabular}{|c|c|c|c|c|c|c|}
\hline \multirow[b]{2}{*}{ Experiment } & \multicolumn{2}{|c|}{ Rehearsed Items } & \multicolumn{2}{|c|}{ Only-Heard Items } & \multicolumn{2}{|c|}{ Lure Items } \\
\hline & $\%$ Yes & Rating & $\%$ Yes & Rating & $\%$ Yes & Rating \\
\hline $\begin{array}{l}1 \text { (Pairs) } \\
2 \text { (Triples) }\end{array}$ & $\begin{array}{l}56.3 \\
53.1\end{array}$ & $\begin{array}{l}3.77 \\
3.60\end{array}$ & $\begin{array}{l}63.5 \\
58.3\end{array}$ & $\begin{array}{l}3.86 \\
3.81\end{array}$ & $\begin{array}{l}39.6 \\
40.6\end{array}$ & $\begin{array}{l}3.15 \\
3.14\end{array}$ \\
\hline
\end{tabular}

\begin{tabular}{|c|c|c|c|c|c|c|}
\hline & \multicolumn{2}{|c|}{ Repeated Items } & \multicolumn{2}{|c|}{ Once-Presented Items } & \multicolumn{2}{|c|}{ Lure Items } \\
\hline & $\%$ Yes & Rating & $\%$ Yes & Rating & $\%$ Yes & Rating \\
\hline 3 (Pairs) & 75.0 & 4.48 & 61.4 & 3.93 & 46.9 & 3.25 \\
\hline
\end{tabular}




\section{EXPERIMENT 2}

Experiments 2 and 3 were run back-to-back with the same subjects; however, they will be reported separately since they were conceived of and treated as two different experiments (and, in fact, each experiment was conducted by a different experimenter) addressing two different questions. The purpose of Experiment 2 was essentially to replicate Experiment 1 and also to increase the complexity of the stimuli with the intention of perhaps providing more cues for discrimination. Thus, in this experiment, triples of tones were used rather than pairs of tones.

\section{Method}

The method was very similar to that of Experiment 1 . The only difference was that the stimuli consisted of three tones played in succession rather than two. In fact, they were created by adding another tone to the pairs used in the previous experiment. The design and procedure were as before.

The subjects were 24 students of The American University who participated in the experiment for extra credit in psychology courses. None reported having any hearing problems or absolute pitch. Of the 24 subjects, 13 had had no musical training, 8 had had at least 2 years of training (ranging from 2 to 12 years, with a mean of 6.9 years), and 3 had had some training but classified themselves as musically naive (for reasons such as no longer remembering how to read music or play an instrument).

\section{Results}

The results (summarized in Table 1) essentially mirrored those of Experiment 1. Overall, there was an effect of presentation; the subjects remembered presented items better than lure items $[t(23)=2.23$, for percentage of yes responses and $t(23)=2.97$ for recognition ratings, both $p s<.025]$. When rehearsed and only-heard items were analyzed separately, the effect of presentation emerged rather weakly for rehearsed items and only for recognition ratings $[t(23)=1.79, p<.05$; the $t$ value for percentage of yes responses was $1.40, p>.10]$. The effect was healthy with only-heard items $[t \mathrm{~s}(23)=2.54$ and 3.29 , ps $<.01$, for percentage of yes responses and recognition ratings, respectively]. The musically trained and naive subjects (including the three uncertain subjects) gave the same patterns of results.

As before, rehearsal did not appear to benefit memory. The subjects did not recognize rehearsed triples better than only-heard triples; in fact, again, only-heard items were slightly, though insignificantly ( $p s>.10$ ), better remembered. There were again no order effects, no correlations between ease of rehearsal ratings at study and actual recognition performance, and no differences in the overall recognition patterns of results of musically trained and naive subjects.

\section{EXPERIMENT 3}

Experiment 3 explored the effect of repetition during presentation on recognition performance, using the materials and design of Experiment 1. In Experiment 1, there was no effect of covert rehearsal. Thus, we were interested in seeing whether experimenter-imposed repetition, a close cousin of rehearsal, would have an effect on memory.

\section{Method}

The method was very similar to that of Experiment 1 . The only differences were in the presentation phase. There were no rehearsal intervals (and hence no ease of rehearsal ratings during study). Each pair of tones was separated from the next one by a 2 -sec interval. Four of the pairs were presented once and the other four twice; each presentation of a repeated pair was separated by at least one other tone pair. Thus, there were 12 token pair presentations. The counterbalancing measures regarding presentation type and target-lure status were the same as in Experiment 1, with repeated items being treated as rehearsed items and once-presented items as only-heard items. One other difference was that unlike in Experiment 1, in which the subjects expected the recognition test to include only the rehearsed items, in this experiment, the subjects knew the test would include all items. They were told to try to remember each item, and though they were told that some items would be repeated, they were not told when a repetition occurred. The subjects were the same as in Experiment 2.

\section{Results}

As can be seen in Table 1, presented items were better recognized than were lure items $[t \mathrm{~s}(23)=2.91$ and 3.65 , ps $<.01$, for percentage of yes responses and recognition ratings, respectively]. Corresponding separate analyses for once-presented items also indicated better recognition for these items than for lure items $[\operatorname{ts}(23)=2.13$ and 2.73, ps $<.025$ ]. Again, the patterns of results for musically trained and naive subjects were not different.

Of main interest was the effect of repetition. Unlike covert rehearsal, repetition benefited memory. Although the difference between twice- and once-presented items failed to reach significance in percentage of yes responses $[t(23)=1.66, p>.10]$, twice-presented items were given reliably higher recognition ratings (a more sensitive measure than percentage of yes responses) than were once-presented items $[t(23)=2.25, p<.05]$. The patterns of results were the same for musically trained and naive subjects.

\section{SUMMARY AND CONCLUSION}

Experiments 1 and 2 showed that covert rehearsal of pairs of tones did not benefit memory beyond simply hearing them. Experiment 3 showed that repetition of those tones, or hearing them twice, on the other hand, did benefit memory compared to hearing them only once. Moreover, although the issue was not systematically manipulated, there appeared to be no differences in the patterns of results from musically trained and musically naive subjects.

Covert rehearsal of auditory aspects of materials is possible (e.g., Watkins \& Peynircioglu, 1982). Also, there is little doubt that overt rehearsal, at least with more complex musical stimuli, does benefit memory. After all, musicians can memorize pieces, and even nonmusicians learn songs and come to recognize pieces with repeated exposures. Our Experiment 3 also showed an advantage of repetition; thus, even memory for such difficult stimuli as pairs of tones can benefit from perceptual repetition. It was, therefore, somewhat surprising that covert rehearsal of tone pairs or triples did not enhance memory compared to simply hearing them. At this stage, one can only speculate why we did not observe any beneficial effects of rehearsal for the tones. One factor could be the lack of different "dimensions" or types of information to mentally manipulate in these tone pairs or triples. Perceptual memory research usually indicates successful immediate memory performance for single-dimensional stimuli such as intensity of taste, smell, or tones 
(e.g., Köhler, 1923; Needham, 1934). But, such memory "fades" within seconds. Had we used more complex musical stimuli, then, with additional perceptual dimensions such as different rhythms, covert rehearsal might, indeed, have enhanced memory. It is nevertheless of interest to see that with simple musical tone pairs, even though repetition enhances memory, covert rehearsal does not.

\section{REFERENCES}

FARAh, M. J., \& SMITH, A. F. (1983). Perceptual interference and facilitation with auditory imagery. Perception \& Psychophysics, 33, 475-478.

Graefe, T. M., \& Watkins, M. J. (1980). Picture rehearsal: An effect of selectively attending to pictures no longer in view. Journal of Experimental Psychology: Human Learning \& Memory, 6, 156-162.

HALPERN, A. R. (1988). Mental scanning in auditory imagery for songs. Journal of Experimental Psychology: Learning, Memory, \& Cognition, 14, 434-443.
Hubbard, T. L., \& Stoeckig, K. (1988). Musical imagery: Generation of tones and chords. Journal of Experimental Psychology: Learning, Memory, \& Cognition, 14, 656-667.

JoHNSON, R. E. (1980). Memory-based rehearsal. In G. Bower (Ed.), The psychology of learning and motivation (Vol. 14, pp. 263-307). New York: Academic Press.

KöHLER, W. (1923). Zur Theorie des Sukzessivvergleichs und der Zeitfehler. Psychologische Forschung, 4, 115-175.

Needham, J. G. (1934). The time error in comparison judgments. Psychological Bulletin, 31, 229-243.

Watkıns, M. J., \& PeynircioğLu, Z. F. (1981). [Rehearsal of natural sounds]. Unpublished raw data.

Watkıns, M. J., \& PeynircioĞlu, Z. F. (1982). A perspective on rehearsal. In G. Bower (Ed.), The psychology of learning and motivation (Vol. 16, pp. 153-190). New York: Academic Press.

(Manuscript received March 13, 1992.)

\section{Third International Conference on Visual Search University of Nottingham August 24-27, 1992}

The Applied Vision Association, the Ergonomics Society, and the British Machine Vision Association and Society for Pattern Recognition are sponsoring the Third International Conference on Visual Search, to be held at the University of Nottingham, U.K., August 24-27, 1992.

This is the third in a series of international conferences devoted exclusively to all aspects of visual search processing. The meeting will encompass both human and machine vision approaches. The sessions will include (but not be limited to): attention and segmentation, eye movements, computer vision, search modeling, and applied aspects of search. There will also be a workshop, and the conference proceedings will be published.

For further information, contact: TICVS, Academic Radiology, University Hospital, Queens Medical Centre, Clifton Boulevard, Nottingham NG7 2UH, U.K. (Tel., 44(0)602 709442; FAX/ANSWERPHONE, 44(0)602 709140). 